\title{
A DRENAGEM PLEURAL PRÉ-HOSPITALAR: APRESENTAÇÃO DE MECANISMO DE VÁLVULA UNIDIRECIONAL
}

\section{PRE-HOSPITAL CHEST DRAINAGE: PRESENTION ONE-WAY VALVE MECHANISM}

\author{
Alexandre Garcia de Lima'; ;van Felizardo Contrera Toro²; \\ Alfio José Tincani, TCBC-SP ${ }^{3}$; Gilson Barreto ${ }^{4}$
}

\begin{abstract}
Resumo: Objetivos: O objetivo do presente estudo é apresentar um mecanismo de válvula unidirecional para substituição do selo de água na drenagem pleural tubular fechada, em ambiente pré-hospitalar, bem como registrar os resultados de seu uso inicial no SAMU-Campinas/SP/Brasil. Método: Foram realizadas 22 (vinte e duas) drenagens pleurais com válvula em doentes vítimas de traumatismo ou pneumotórax espontâneo, todos em ambiente pré-hospitalar, de forma prospectiva, não randomizada. Resultados: $\mathrm{O}$ débito total de líquidos através da válvula variou de zero a $1500 \mathrm{ml}$, com média de $700 \pm 87,4 \mathrm{ml}$, para um tempo de percurso em média de $18 \pm 1,1$ minutos, variando de 8 a 26 minutos. A frequência cardíaca inicial foi $120 \pm 2,7$ bpm e final de $100 \pm 2$ bpm (p 0,00) e a frequência respiratória inicial foi $24 \pm 0,8 \mathrm{ipm}$ e o valor final foi de $15 \pm 0,3$ ipm (p 0,03). Houve apenas duas falhas mecânicas do sistema e uma foi corrigida pela substituição da mesma, trazudindo num índice de sucesso de 95,4\% neste trabalho. Conclusões: Levando em conta exame físico inicial com o exame físico final, bem como pela quantificação de débitos, concluímos que a válvula mostrou-se eficiente e funcionante, e que é segura para o uso em urgências pré-hospitalares (Rev. Col. Bras. Cir. 2006; 33(2): 101-106).
\end{abstract}

Descritores:: Drenagem/instrumentação; Serviços médicos de emergência; Traumatismos torácicos.

\section{INTRODUÇÃO}

A implantação nacional dos Serviços de Atendimentos Móveis de Urgência, ou SAMU-192, levou médicos emergencistas e intervencionistas para o local do atendimento inicial, abreviando o tempo para o início do tratamento de suporte e por vezes mesmo definitivo de doentes graves e agudamente enfermos.

No entanto, várias peculiaridades do atendimento pré-hospitalar têm feito os médicos buscarem novas alternativas aos bem estabelecidos mecanismos e condutas da sala de urgência de um hospital ou pronto socorro. Dentro deste cenário de intervenção precoce encontra-se a drenagem pleural em ambiente pré-hospitalar. O selo de água para a drenagem pleural, mecanismo de fluxo unidirecional mais frequentemente usado, ${ }^{1,2}$ é incoveniente e inseguro para a drenagem préhospitalar, ${ }^{3,4}$ pois não há espaço para colocação segura do frasco, o desnivelamento do frasco em relação ao doente é insuficiente e são necessários frequentes oclusões temporárias do sistema através de clampe, procedimento proscrito e potencialmente fatal.

Apresenta-se neste trabalho um mecanismo de válvula unidirecional para substituição do selo de água convencional, denominado Dispositivo de Válvula Unidirecional DVU, concebido, desenvolvido e fabricado na Universidade
Estadual de Campinas - UNICAMP, através do Departamento de Engenharia Biomédica e do Centro de Tecnologia desta Universidade. Este mecanismo recebeu menção honrosa pelo mérito de invento do Prêmio Governador do Estado de São Paulo em 1992 com os inventores Alfio José Tincani e Gilson Barreto.

Foi previamente usada em doentes com pneumotórax espontâneo que deram entrada no Pronto Socorro do Hospital das Clínicas da Unicamp. Nesta ocasião foram instalados oito DVU, com resolução completa de todos os casos, dados apresentados no XII Congresso Brasileiro de Cirurgia Torácica - Tórax 2001, com o título "Válvula unidirecional para o dreno de tórax - alternativa para o selo de água".

\section{MÉTODO}

A válvula aqui apresentada (Figura 1) consiste de um frasco de dreno de acrílico polido de 02 " $x 90 \mathrm{~mm}$ que possui na sua extremidade mais larga uma rosca para conexão com a tampa do frasco, também de acrílico polido de 02"x $90 \mathrm{~mm}$, onde se interpõe o mecanismo valvular maleável, de molde rígido de borracha natural de látex. À tampa do frasco se conecta o dreno tubular multifenestrado 38 French de diâmetro de borracha maleável que foi previamente introduzido na cavidade pleural pela técnica de dissecção romba. Na extremi-

1. Médico Cirurgião Torácico; Aluno de Mestrado da Faculdade de Ciências Médicas da Unicamp; Médico Emergencista do Serviço de Atendimento Móvel de Urgência (SAMU-192) de Campinas; Mestrando da Pós-Graduação em Cirurgia da Faculdade de Ciências Médicas da Unicamp.

2. Médico Cirurgião Torácico; Chefe da Disciplina de Cirurgia Torácica do Hospital das Clínicas da Unicamp.

3. Médico Cirurgião de Cabeça e Pescoço; Professor Assistente da Disciplina de Cirurgia de Cabeça e Pescoço do Hospital das Clínicas da Unicamp.

4. Médico Cirurgião de Cabeça e Pescoço; Chefe do Serviço de Oncologia do Hospital Municipal de Paulínia.

Recebido em 20/10/2005

Aceito para publicação em 22/12/2005

Conflito de intersses: nenhum

Fonte de financiamento: nenhuma

Trabalho realizado no Serviço de Atendimento Móvel de Urgência (SAMU-192) de Campinas e no Hospital das Clínicas da UNICAMP - SP 


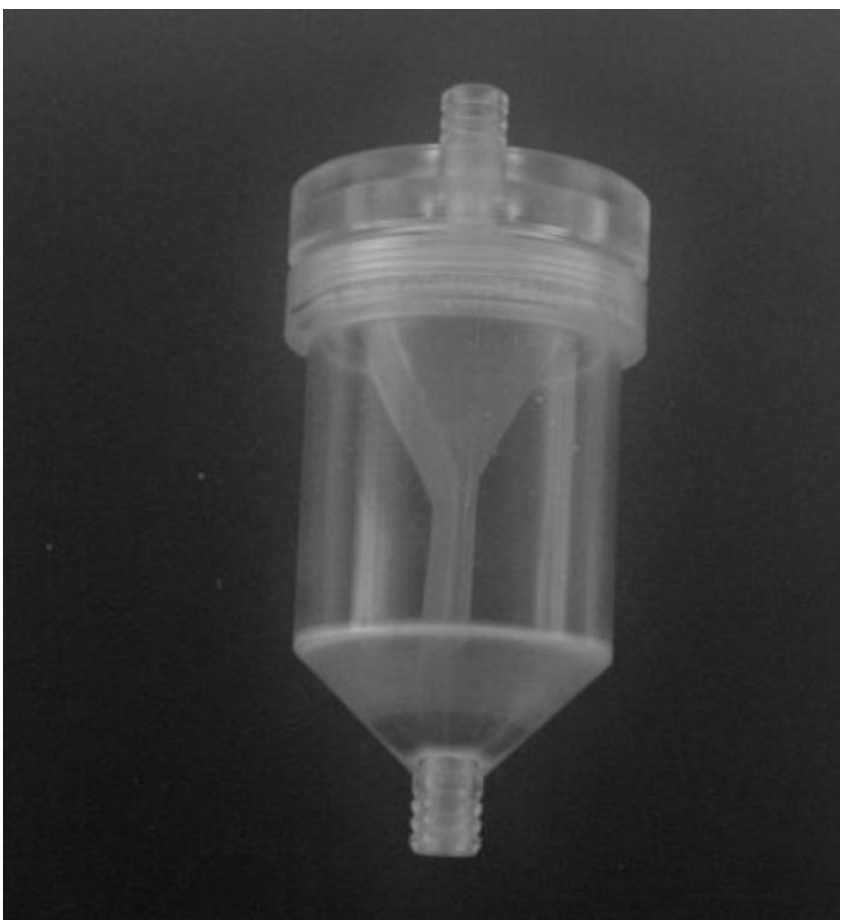

Figura 1 - Válvula unidirecional para drenagem pleural tubular fechada.

dade distal do frasco do dreno há conexão para o sistema coletor tradicional.

A válvula foi testada pelo Centro de Tecnologia da Unicamp através do Laboratório de Metrologia através de balança de pressão Budenberg modelo 450 ar/gás, número de série 2531/45, pistão número de série A-4367, faixa de indicação 15 a 1000 mbar, certificado de calibração n ${ }^{\circ}$ DIMCI - 0015/ 2002. O teste foi realizado comparando-se o padrão do laboratório com as amostras de válvulas conectadas à balança de pressão, sendo produzido vácuo através de um dispositivo no sistema da balança. Foi utilizada a menor massa do laboratório, de 15 mbar. Todas as válvulas testadas fecharam-se com a pressão de 15 mbar (aproximadamente $0,013 \mathrm{~cm} \mathrm{H}_{2} 0$ ).

Em se tratando de traumatismo torácico penetrante ou fechado e em casos de pneumotórax espontâneo, após medidas de estabilização iniciais segundo normas do Comitê de Trauma do Colégio Americano de Cirurgiões (Advanced Trauma Life Support Program for Doctors, 6a . edição, 1997), era realizada a drenagem tubular pleural fechada com antissepsia adequada, anestesia local e técnica de drenagem romba, com exploração digital da cavidade pleural. Após a inserção do dreno pleural conectava-se o mecanismo valvular à extremidade distal do dreno, e então este era conectado ao frasco coletor para que débitos líquidos e escape aéreo pudessem ser notados logo após a drenagem e durante o transporte.

Além desses parâmetros, foram anotados dados hemodinâmicos como frequência cardíaca, frequência respiratória, pressão arterial com manguito de mercúrio, quantidade e tipo de volume endovenoso infundido no período pré-hospitalar, necessidade de procedimentos adicionais como intubação translaríngea, cricotireoidostomia cirúrgica e flebotomia. O exame semiológico completo e principalmente a ausculta e percussão torácicas, inspeção da respiração e de turgência jugular, foram a base de sustentação para a decisão do autor, conforme sustenta a literatura, ${ }^{5-12}$ para a realização ou não da drenagem pleural naqueles pacientes vítimas de traumatismo fechado ou naqueles em que se suspeitou de pneumotórax espontâneo e que não tinham comprovação radiológica. Todos parâmetros previamente descritos eram reavaliados ao final do atendimento pré-hospitalar no momento da chegada ao pronto socorro hospitalar.

Os doentes eram identificados por sexo, idade e raça. Os dados foram anotados em formulário previamente concebido e submetido ao Comitê de Ética em Pesquisa da Faculdade de Ciências Médicas da Universidade Estadual de Campinas, e foram informatizados através do software EPI-INFO 6, com o qual foram feitas as análises estatísticas.

O termo de consentimento foi dispensado por se tratar de situação de urgência e risco iminente de morte e situações em que o indivíduo tem limitada capacidade de decisão devido suas condições clínicas, conforme parecer projeto de número 329/2002 do Comitê de Ética em Pesquisa da Faculdade de Ciências Médicas da Universidade Estadual de Campinas.

\section{RESULTADOS}

Foram colocadas, no período compreendido entre maio de 2002 e maio de 2004, um total de 22 válvulas em pacientes submetidos à drenagem pleural tubular, todos eles atendidos pelo autor.

Dos 21 doentes atendidos, 19 (90,5\%) eram do sexo masculino. A média de idade foi de $25 \pm 2,16$ anos, variando entre 13 e 45 anos.

Quanto à origem dos chamados, 13 (61,9\%) foram atendidos em via pública e 8 (38,1\%) foram atendidos em Pronto-atendimentos em Saúde.

A drenagem pleural tubular com válvula foi realizada em $16(72,7 \%)$ pacientes com ferimentos penetrantes da caixa torácica, sendo que destes, cinco $(22,7 \%)$ por ferimento por arma branca e 11 (50\%) por ferimento por projétil de arma de fogo. Três $(13,6 \%)$ pacientes foram submetidos à drenagem devido a traumatismo torácico fechado com alteração na ausculta e/ou percussão pulmonar, e outros três (13,6\%) por pneumotórax espontâneo com repercussão clínica.

No momento do atendimento inicial, 21 (95,5\%) tinham alteração da ausculta pulmonar e/ou da percussão torácica, principais indicadores para a drenagem pleural. $\mathrm{O}$ único paciente que não tinha alteração nestes dois parâmetros foi previamente drenado em um Pronto Atendimento devido a um pneumotórax espontâneo.

Treze pacientes foram inicialmente atendidos em locais públicos e foram vítimas de ferimento por arma branca em quatro casos (30,8\%), por ferimento por projétil de arma de fogo em seis casos (46,2\%) e por trauma fechado em três $(23,1 \%)$ casos. Todas as vítimas de traumatismo fechado torácico submetidas à drenagem apresentavam movimento paradoxal durante o ciclo respiratório da parede torácica, caracterizando tórax instável.

A quantificação do débito do momento da partida da viatura do local do atendimento inicial até o destino final do 


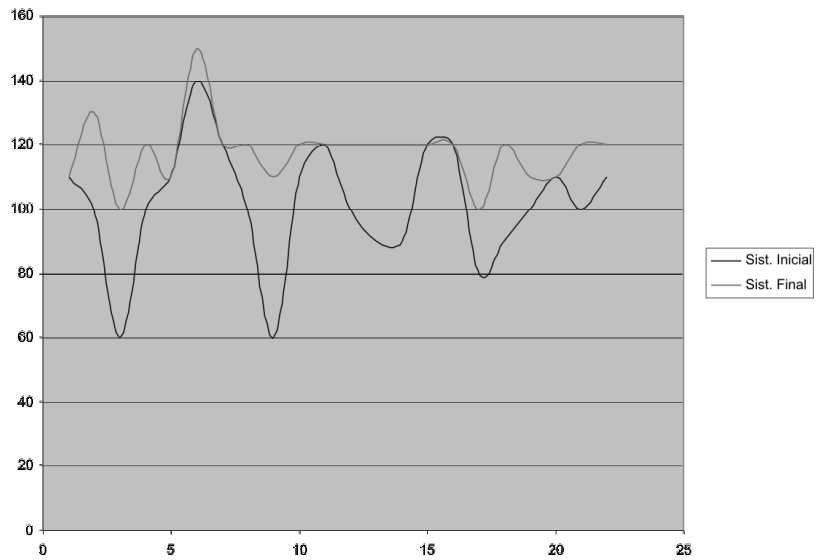

Figura 2 - Comparação dos indivíduos quanto a pressão arterial sistêmica sistólica inicial e final.

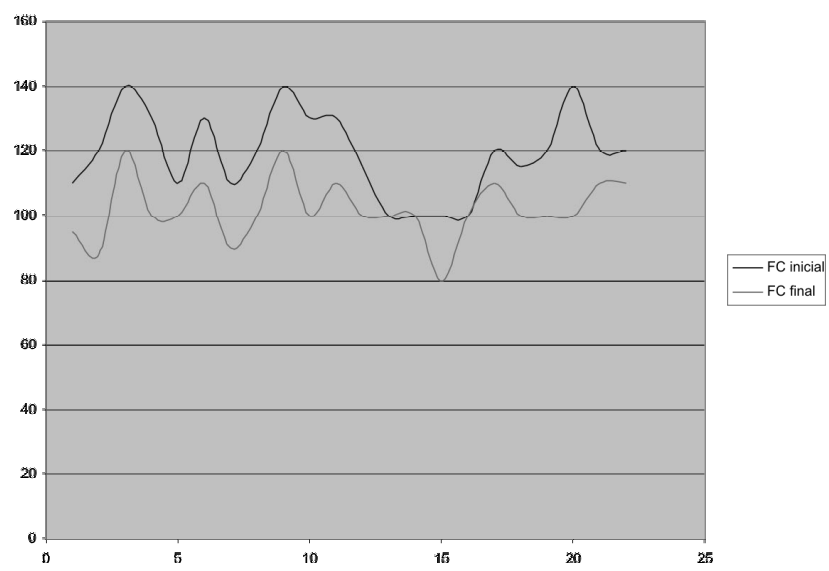

Figura 3 - Comparação dos indivíduos quanto à freqüência cardíaca inicial e final.

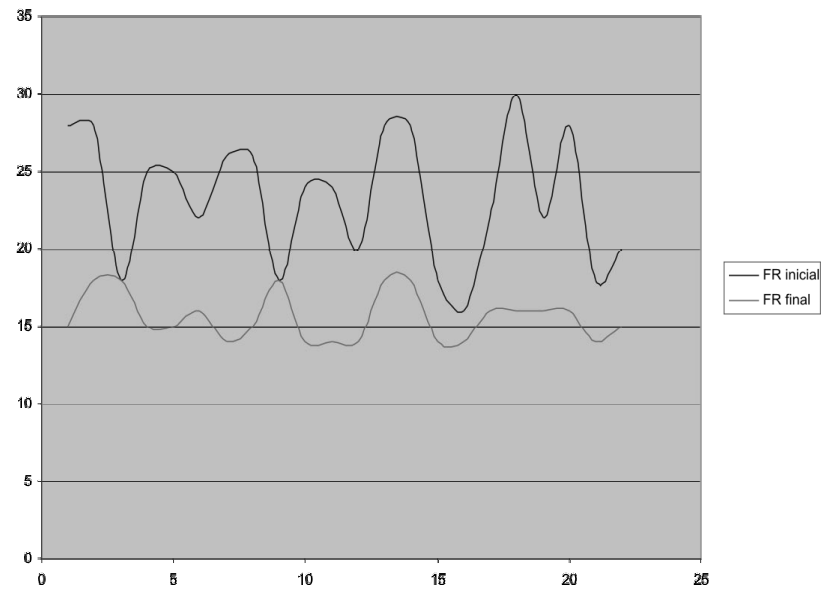

Figura 4 - Comparação dos indivíduos quanto à freqüência respiratória inicial e final.

doente variou de zero a $500 \mathrm{ml}$, com média de $200( \pm 36,1) \mathrm{ml}$. O tempo gasto para realização deste percurso foi em média de 18 $( \pm 1,1)$ minutos, variando de 8 a 26 minutos.

O débito total de líquidos através da válvula, ou seja, o débito inicial somado ao débito durante o percurso da viatura até o destino final variou de zero a $1500 \mathrm{ml}$, com média de $700( \pm 87,4) \mathrm{ml}$.
Levando agora em consideração todos doentes atendidos, nove apresentavam escape aéreo inicial, e sete apresentavam enfisema do tecido celular subcutâneo à palpação da parede torácica. Entre aqueles que tinham enfisema subcutâneo, quatro também tiveram escape aéreo. Nenhum desenvolveu ou aumentou o enfisema subcutâneo durante o transporte.

A pressão arterial sistólica inicial variou entre 60 mmHg e $140 \mathrm{mmHg}$, com média de $100( \pm 4) \mathrm{mmHg}$, sendo que seis $(27,3 \%)$ doentes apresentavam sistólica inicial menor ou igual a $90 \mathrm{mmHg}$, caracterizando-se choque hemorrágico grau II.

Comparando-se os parâmetros hemodinâmicos como pressão sistólica e freqüência cardíaca, bem como a freqüência respiratória de todos os pacientes incluídos neste estudo, desde a abordagem inicial até a chegada ao hospital e a transferência aos plantonistas, temos como a média inicial pressão arterial sistólica $100( \pm 4) \mathrm{mmHg}$ e pressão arterial sistólica final de $120( \pm 2,1) \mathrm{mmHg}(p 0,00)$, (Figura 2), como média da frequência cardíaca (Figura 3) a inicial $120( \pm 2,7) \mathrm{bpm}$ e final de $100( \pm 2) \mathrm{bpm}(p 0,00)$ e levando em conta a frequência respiratória, o valor inicial da média foi $24(0,8)$ ipm e o valor final foi de $15( \pm 0,3)$ ipm ( $p$ 0,03), (Figura 4).

Houve uma (7,7\%) falha mecânica do sistema neste subgrupo, onde a válvula inicialmente usada perdeu a vedação na rosca e foi prontamente substituída. A outra falha não ocorreu neste subgrupo mas sim em um paciente previamente drenado no Pronto Atendimento por pneumotórax espontâneo, onde durante a tentativa de acoplamento do DVU notou-se incompatibilidade de diâmetros entre o dreno torácico usado e o encaixe da parte proximal da válvula, neste caso foi possível a substituição da válvula.

\section{DISCUSSÃO}

O traumatismo torácico é responsável por 25\% dos óbitos nas vítimas de trauma em geral, e $12 \%$ dos traumatismos torácicos isolados não sobrevivem, ${ }^{13}$ muitas vezes estando associados a traumatismos de outros segmentos corporais e aumentando assim a complexidade do atendimento. ${ }^{14,15}$. A mortalidade total nos EUA em 1982 foi de 462,3:100.000 habitantes e destes, 36,6 óbitos foram devidos a acidentes e suas conseqüências; houve 12.000 homicídios por armas de fogo ${ }^{16}$. Já em 1985 ocorrem 94.000 mortes por acidentes nos EUA, significando 40 mortes para cada 100.000 habitantes, sendo que os traumatismos torácicos responderam por $20 \%$ dos óbitos de uma forma geral e em $2 / 3$ das vezes estavam associados a acidentes automobilísticos ${ }^{17}$. A maior parte dos doentes vítimas de traumatismo torácico agudo é tratada com drenagem pleural tubular fechada e somente $15 \%$ destes doentes necessitam de tratamento cirúrgico no tórax propriamente dito $^{14,17,18,19}$. No período de oito anos da guerra do Irã-Iraque houve 173.823 admissões hospitalares nos serviços de campo do Irã, sendo que destes, 32534 doentes foram submetidos a algum tipo de intervenção cirúrgica; a segunda intervenção mais freqüente (18,3\% das vezes) foi a drenagem torácica, ficando depois apenas da laparotomia exploradora ${ }^{20}$. 
O tratamento pré-hospitalar vem melhorando a sobrevida global de todos os doentes vítimas de traumatismos de quaisquer segmentos corporais, sendo que doentes críticos vítimas de ferimentos penetrantes do tórax e/ou no abdome tratados no Hospital Geral de Denver, Colorado, EUA, tiveram uma sobrevida global de 81,8\%, sobrevida e alta hospitalar em 94\% dos que tinham pressão arterial inaudível com manômetro de mercúrio e sobrevida de 18,2\% dos que não tinham nenhum sinal vital, no momento da chegada da ambulância ${ }^{21}$.

O tratamento do paciente, com traumatismo torácico, no ambiente pré-hospitalar é fator de discussão e de teorias conflitantes, no que tange à diferenciação do que deve ser feito no pré-hospitalar e no intra-hospitalar ${ }^{6,11,22}$.

A drenagem pleural, no entanto, quando realizada com técnica correta, tanto cirúrgica quanto de anti-sepsia, temse mostrado um procedimento seguro, rápido e efetivo, com grande benefício aos doentes. Millikan et al. ${ }^{23}$ demonstraram em 447 drenagens pleurais devido a traumatismo torácico agudo somente $1 \%$ de complicações (duas lesões diafragmáticas, uma lesão pulmonar e um tubo intraabdominal) e índice de empiema de 2,4\% (variando na literatura de 1 a $16 \%$ de incidência, sendo na maior parte das séries em torno de 3\%, índice este considerável como aceitável em centros de atendimento ao traumatismo).

Paralelamente à questão do drenar ou não drenar no ambiente pré-hospitalar, surge outro problema a ser resolvido: a questão do selo de água na drenagem pleural.

A drenagem pleural fechada subaquática ou em selo de água, descrita inicialmente por Playfair em $1872^{24,25}$ e posteriormente modificada por Kenyon de Nova Iorque em 1916, ${ }^{25}$ sistema de fluxo unidirecional mais comumente usado, têm-se mostrado inseguro no ambiente pré-hospitalar, pois há dificuldade para mantê-los desnivelados em relação ao doente dentro do exíguo espaço dentro da ambulância e usualmente são clampeados, o que representa um risco em potencial, principalmente naqueles doentes em que haja um escape aéreo de grande volume $e^{1,3,4,26}$.

Alguns mecanismos alternativos que permitam o fluxo unidirecional necessário à reexpansão pulmonar, isto é, permitam a saída de líquidos e gases durante a expiração e impeçam o refluxo destes para a cavidade pleural durante a inspiração, têm sido descritos na literatura.

O mecanismo alternativo mais comumente usado em substituição ao selo de água é a válvula de Heimlich²,28,29,30. Esta válvula, descrita inicialmente para o traumatismo torácico dos soldados norte-americanos nos campos de batalha das guerras do Vietnã e de Israel, é prática, barata e pequena, ${ }^{31}$ tem sido colocada em prática em diversos serviços pré-hospitalares e hospitalares ${ }^{32}$ e mesmo no âmbito nacional, ${ }^{33}$ mas não é livre de complicações. Não deve ser usada na presença de sangue ou fluidos pleurais, pois tanto a extremidade proximal quanto a distal podem ocluir ${ }^{34}$, complicação esta sanada pelo DVU através do desenho da válvula de látex, que ao contrário do mecanismo da válvula de Heimlich, que é flácido e com baixa capacidade de manter a expansão pulmonar na presença de grandes escapes aéreos ou na presença de líquidos, é rígida e não sofre oclusão por fluidos. Além disso a válvula de
Heimlich possui simetria radial e portanto é difícil diferenciar a extremidade distal da proximal, sendo relatado mesmo caso de pneumotórax hipertensivo por conexão reversa da válvula ${ }^{35}$. Apesar de ser um mecanismo disponível no mercado nacional, seu custo para aquisição ainda é elevado (no mercado norte-americano pode ser adquirida por U\$ 15, mas no Brasil o seu custo médio é de R\$900,00) e possui os inconvenientes descritos.

Visto o exíguo conhecimento médico de uma forma geral no Brasil quanto a mecanismos de substituição ao tradicional selo de água e também à dificuldade de acesso dos médicos emergencistas a estas válvulas, e mesmo devido ao custo de importação das mesmas, e, paralelamente, a necessidade de adequação do tratamento do traumatismo torácico em ambiente pré-hospitalar no Brasil, testou-se o Dispositivo de Válvula Unidirecional para a drenagem pleural tubular fechada, no contexto do atendimento préhospitalar realizado no SAMU-192 de Campinas, São Paulo, Brasil.

O DVU descrito possui uma diferença básica que o torna mais seguro em relação à válvula de Heimlich, que é a capacidade de manter-se pérvio na presença de líquidos, devido ao seu molde rígido de borracha natural de látex.

A primeira preocupação do estudo foi demonstrar, através da quantificação dos débitos e através da comparação entre parâmetros hemodinâmicos, respiratórios e também dos exames físicos dos doentes como ausculta pulmonar e cardíaca e percussão torácica, se o mecanismo demonstrava ser seguro para o uso na urgência e dentro de ambulâncias.

Mas talvez os parâmetros mais importantes para assegurar a eficiência tanto da drenagem torácica com o DVU quanto das manobras iniciais ressuscitativas e de suporte avançado de vida sejam os dados dos exames clínicos do doente traumatizado.

Quanto à ausculta pulmonar e percussão pulmonar, inicialmente, levando em conta todo o grupo, temos que seis $(27,3 \%)$ tinham murmúrio vesicular diminuído com percussão torácica normal, cinco (22,7\%) apresentavam murmúrio também diminuído porém com percussão hipertimpânica e 10 (45,5\%) doentes tinham murmúrio diminuído com percussão submaciça; um doente (4,5\%) apresentava ausculta pulmonar e percussão torácica normais, e tinha sido previamente drenado por pneumotórax espontâneo. A propedêutica pulmonar à chegada aos hospitais deste grupo total de doentes apresentava-se da seguinte forma: três (13,6\%) casos com murmúrio vesicular diminuído porém com percussão torácica normal, e nos outro 19 casos $(86,4 \%)$ tanto a ausculta quanto a percussão estavam inalteradas.

Somado ao fato de que houve melhora na freqüência cardíaca, freqüência respiratória e pressão arterial sistêmica, fatos esses onde estão envolvidos outros fatores que não a drenagem somente, como reposição volêmica, estabilização de vias aéreas, imobilização de fraturas, suporte com oxigenioterapia e suporte emocional para as vítimas, concluise que o atendimento pré-hospitalar realizado de forma global, simultânea e eficiente tem grande repercussão no tratamento integral desses doentes. 
Nota-se que será de grande valia para o sistema de saúde de uma forma geral, e mais especificamente, no atendimento de urgências, a ampla divulgação de mecanismos como este, o que gerará discussões sobre condutas no traumatismo torácico e no doente politraumatizado como um todo, podendo inclusive ser instalada uma política de treinamento dessas equipes.

Levando em conta que houve melhora clínica através da propedêutica pulmonar/torácica, comparando-se o exame físico inicial com o exame físico final no momento da passagem de caso no Hospital, conclui-se que a válvula mostrou-se eficiente e funcionante, e que é segura para o uso em urgências pré-hospitalares. Houve apenas dois casos de falha no sistema, e em um desses casos, a válvula defeituosa foi substituída prontamente por outra, com bom funcionamento; no outro caso, devido a incompatibilidade de diâmetros entre o dreno utilizado e o DVU, não foi possível o uso do sistema. Considerando apenas este último caso como falha definitiva, temos um índice de eficiência de $95,4 \%$.

\begin{abstract}
Background: The purpose of this study is to present an one-way valve mechanism to replace the underwater seal for pleural drainage, currently used in the prehospital attendance, as well as document preliminary results of its initial use at SAMUCampinas/SP/Brasil. Methods: Twenty two pleural drains with the valve were carried out, all of these in prehospital environment, in patients who suffered thoracic trauma or spontaneous pneumothorax, in a prospective not randomized trial. Results: The total volume output drained through the valve ranged from 0 to $1500 \mathrm{ml}$, mean $700 \pm 87,4 \mathrm{ml}$, in an average time lag of 18 minutes ( $\pm 1,1$ minutes - ranging from 8 to 26 minutes). The initial and final cardiac frequency was $120 \pm 2,7 \mathrm{bpm}$ and $100 \pm$ $2 \mathrm{bpm}(p 0,00)$, respectively, whereas the initial respiratory frequency was $24 \pm 0,8 \mathrm{ipm}$ and the final was $15 \pm 0,3 \mathrm{ipm}(p$ $0,03)$. Only two mechanical failures were registered, one of which was corrected by the substitution of the defective valve, showing a 95,4\% success ratio. Conclusions: Taking into account the initial and final physical examination, as well as the output quantification, it can be concluded that the valve is efficient and functional, and safe in the pre-hospital emergencies.
\end{abstract}

Key words: Drainage/instrumentation; Emergency medical services; Thoracic injuries.

\section{REFERÊNCIAS}

1- Hyde J, Sykes T, Graham T. Reducing morbidity from chest drains. BMJ. 1997; 314(7085):914-5.

2- McMahon-Parkes K. Management of pleural drains. Nurs Times. 1997; 93(52):48-9

3- Graham AN, Cosgrove AP, Gibbons JRP, McGuigan JA. Randomised clinical trial of chest drainage systems. Thorax.1992; 47(6):461-2.

4- Adegboye VO, Adebo OA, Osinowo O, Brimmon JA. Closet chest drainage without an underwater seal. Afr J Med Med Sci. 1996; 25(4):353-5

5- Baldt MM, Bankier AA, Germann PS, Pöschl GP, Skrbensky GT, Herold CJ. Complications after emergency tube thoracostomy: assessment with CT. Radiology. 1995; 195(2):539-43.

6- Barton ED, Epperson M, Hoyt DB, Fortlage D, Rosen P. Prehospital needle aspiration and tube thoracostomy in trauma victims: a six-year experience with aeromedical crews. J Emerg Med. 1995; 13(2):155-63.

7- Carli P, Lejay M. Réanimation initiale dês traumatismes fermés du thorax. Rev Prat. 1997; 47:951-7.

8- Coats TJ, Wilson AW, Xeropotamous N. Pre-hospital management of patients with severe thoracic injury. Injury 1995; 26(9):581-5.

9- Demartines N, Kiener A, Scheidegger D, Harder F. Drainage thoracique sur les lieux d'accidents. Helv Chir Acta. 1990; 57:2737.

10- Schmidt U, Stalp M, Gerich T, Blauth M, Maull KI, Tscherne H. Chest tube decompression of blunt chest injuries by physicians in the field: effectiveness and complications. J Trauma.1998; 44(1):98-101.

11- Wayne MR, McSwain NE, Jr. Clinical evaluation of a new device for the treatment of tension pneumothorax. Ann Surg. 1980; 191(6):760-2.
12- Saad Jr R, Dorgan Neto V, Aguiar Neto JR, Rasslan S. Traumatismos torácicos: sistematização na avaliação inicial. Arq Méd Hosp Fac Ciênc Méd Santa Casa São Paulo.1989; 9(35/ 36):70-2.

13- Botter M, Saad Jr R, Giannini JA, Dorgan Neto V. Drenagem pleural no trauma torácico. J Pneumol. 1996; 22(2):59-64.

14- Machado FK, Jaenisch Neto JB, Tavares LA, Kae MM. Trauma de tórax: experiências com 48 casos. Momento \& Perspectiv Saúde. 1990; 4(1/2):61-3.

15- Baker CC. Epidemiology of trauma: the civilian perspective. Ann Emerg Med. 1986; 15(12):1389-91.

16- LoCicero J $3^{\text {rd }}$, Mattox KL. Epidemiology of chest trauma. Surg Clin North Am. 1989; 69(1):15-9.

17- Eddy AC, Luna GK, Copass M. Empyema thoracis in patients undergoing emergent closed tube thoracostomy for thoracic trauma. Am J Surg. 1989; 157(5):494-7.

18- Silas MG, Belluzzo GR, Miguel EJM, Bahdur R, Pires AC. Traumatismos torácicos: análise de 231 casos. Arq Med ABC. 1990; 13(1-2):19-21.

19- Heidarpour A, Jahani MR, Dabbagh A, Khatami MS. Surgical interventions at field hospitals during the Iran and Iraq War. Mil Med. 1999; 164(2):136-7.

20- Pons PT, Honigman B, Moore EE, Rosen P, Antuna B, Dernocoeur J. Prehospital advanced trauma life support for critical penetrating wounds to the thorax and abdomen. J Trauma. 1985; 25(9):82832.

21- Regel G, Stalp M, Lehmann U, Seekamp A. Prehospital care, importance of early intervention on outcome. Acta Anaesthesiol Scand Suppl.1997; 110:71-6.

22- Millikan JS, Moore EE, Steiner E, Aragon GE, Van Way CW $3^{\text {rd }}$. Complications of tube thoracostomy for acute trauma. Am J Surg. 1980; 140(6):738-41.

23- Miller KS, Sahn SA. Chest tubes. Indications, technique, management and complications. Chest. 1987; 91(2):258-64. 
24- Wagner RB, Slivko B. Highlights of the history of nonpenetrating chest trauma. Surg Clin North Am. 1989; 69(1):1-14.

25- Symbas PN. Chest drainage tubes. Surg Clin North Am. 1989; 69(1):41-6.

26- Heimlich HJ. Chest drainage valve. Emerg Med Serv. 1978; 7(3):89-90.

27- Heimlich HJ. Flutter valve to replace underwater drainage bottles. Hosp Top. 1965; 43(1):122-3.

28- Heimlich HJ. Heimlich valve for chest drainage. Med Instrum. 1983; 17(1):29-31.

29- Heimlich HJ. Valve drainage of the pleural cavity. Dis Chest. 1968; 53(3):282-7.

30- Cannon WB, Mark JB, Jamplis RW. Pneumothorax: a therapeutic update. Am J Surg. 1981; 142(1):26-9.

31- Ponn RB, Silverman HJ, Federico JA. Outpatient chest tube management. Ann Thorac Surg. 1997; 64(1):1437-40.

32- Ortega HAV, Lima MP, Denadai JO. Válvula unidirecional aplicada ao tratamento ambulatorial do pneumotórax. J Pneumol. 1996; 22(4):177-80.
33- Connor PA. When and how do you use a Heimlich flutter valve? Am J Nurs. 1987; 87(3):288-90

34-Spouge AR, Thomas HA. Tension pneumothorax after reversal of a Heimlich valve. AJR Am J Roentgenol. 1992; 158(4):763-4.

Como citar este artigo:

Lima AG, Contrera Toro IF, Tincani AJ, Barreto G. A drenagem pleural pré-hospitalar: apresentação de mecanismo de válvula unidirecional. Rev Col Bras Cir. [serial on Internet] 2006 MarApr;33(2). Available from URL: http://www.scielo.br/rcbc

Endereço para correspondência: Alexandre Garcia de Lima Rua João Simões da Fonseca, 70 Cond. Residencial Barão do Café IV 13085-050 - Campinas - SP 